\title{
Multicolored Electrochromism in Polymers: Structures and Devices
}

\author{
Avni A. Argun, ${ }^{\dagger}$ Pierre-Henri Aubert, ${ }^{\dagger}$ Barry C. Thompson ${ }^{\dagger}$ \\ Irina Schwendeman,${ }^{\dagger}$ Carleton L. Gaupp, ${ }^{\dagger}$ Jungseek Hwang,,$\stackrel{\dagger}{\dagger}$ Nicholas J. Pinto, ${ }^{\S}$ \\ David B. Tanner, ${ }^{\ddagger}$ Alan G. MacDiarmid,,,$\|$ and John R. Reynolds ${ }^{*, \dagger}$ \\ Departments of Chemistry and Physics, Center for Macromolecular Science and Engineering, \\ University of Florida, Gainesville, Florida 32611, Department of Chemistry, \\ University of Pennsylvania, Philadelphia, Pennsylvania 19104, and Department of Chemistry, \\ University of Texas at Dallas, Richardson, Texas 75083
}

Received March 1, 2004. Revised Manuscript Received May 12, 2004

\begin{abstract}
A review of electrochromic (EC) polymers and their applications in absorption/transmission, reflective, and patterned electrochromic devices (ECDs) is presented. Fundamental properties of EC materials such as optical contrast, coloration efficiency, switching speed, and stability are described along with the commonly used characterization methods. The origin of electrochromism in conjugated polymers is explained in terms of the electronic structure changes in the backbone upon doping/dedoping. The ability to tailor the EC properties of conjugated polymers and tune their color states via modification of the polymer structure is demonstrated. Multicolor electrochromic materials can be obtained by substitution of a parent polymer and controlled polymerization of comonomers and with blends and laminates of homopolymers. Absorption/transmission-type ECDs from complementarily colored polymers and reflective-type ECDs on metalized substrates are illustrated with several examples from the literature. Finally, several patterning methods that are promising for ECD applications are discussed. Examples of ECDs constructed from patterned electrodes using line-patterning, screen-printing, and metal vapor deposition techniques are investigated for their possible use in commercial applications.
\end{abstract}

\section{Introduction}

Electrochromism is broadly defined as a reversible optical change in a material induced by an external voltage, with many inorganic and organic species showing electrochromism throughout the electromagnetic spectrum. ${ }^{1}$ Suggested theoretically by J. R. Platt ${ }^{2}$ in 1961, the first examples of electrochromic materials and devices were demonstrated by Deb et al. ${ }^{3}$ when he started to work on amorphous and crystalline metal oxides at Cyanamid Corp. Among electrochromic (EC) materials, transition-metal oxides, especially the highband-gap semiconductor tungsten oxide, $\mathrm{WO}_{3}$, have received extensive attention over the past 30 years. ${ }^{4}$ Thin films of amorphous or polycrystalline $\mathrm{WO}_{3}$ can be prepared by vacuum evaporation, reactive sputtering, and sol-gel methods. Initially transparent in the visible region, cation intercalation (reduction) of $\mathrm{WO}_{3}$ to $\mathrm{M}_{x} \mathrm{WO}_{3}$ (M can be hydrogen or an alkali metal) leads to strong absorption bands in the visible region, making it a cathodically coloring material. Many other inorganic materials have been studied for their electrochromic properties such as Prussian Blue, oxides of $\mathrm{V}, \mathrm{Mo}, \mathrm{Nb}$,

* To whom correspondence should be addressed. Phone: (352) 3929151. Fax: (352) 392-9741. E-mail: reynolds@chem.ufl.edu. URL: http://www.chem.ufl.edu/ reynolds.

${ }^{\dagger}$ Department of Chemistry, University of Florida.

$\$$ Department of Physics, University of Florida.

$\S$ University of Pennsylvania.

" University of Texas at Dallas. and $\mathrm{Ti}$ (cathodically coloring), and oxides of $\mathrm{Ni}, \mathrm{Co}$, and Ir (anodically coloring) ${ }^{5}$

Other EC materials include organic small molecules, such as the bipyridiliums (viologens), which are a class of materials that are transparent in the stable dicationic state. Upon one-electron reduction, a highly colored and exceptionally stable radical cation is formed. Thin film electrochromism is observed for polyviologens and Nsubstituted viologens such as heptyl viologen. ${ }^{6}$ More recently, composite systems, where organic molecules are adsorbed on mesoporous nanoparticles of doped metal oxides, have shown improved electrochromic properties. $^{7}$

Conjugated polymers are a third class of EC materials that have gained popularity due to their ease of processability, rapid response times, and high optical contrasts and the ability to modify their structure to create multicolor electrochromes. Of the conjugated EC polymers, derivatives of poly(thiophene) (PTh), poly(pyrrole) (PPy), and poly(aniline) (PANI) are widely studied. ${ }^{8}$ The mechanism of the EC effect and color control will be discussed in detail for conjugated polymers later.

The most common applications of EC materials include a variety of displays, smart windows, optical shutters, and mirror devices. Companies such as the Gentex Corp. and Donnelly have commercialized electrochromic mirrors as rearview visors for the automobile industry. Prototypes of "smart windows" for buildings 
based on inorganic metal oxides have been developed, but often suffer from low cost-effectiveness. Pilkington introduced its first commercial electrochromic smart window product on glass in late 1998, while Sage Electrochromics has developed a switchable (electronically tintable) window called SageGlass. Additionally, researchers from Lawrence Berkeley Laboratories have installed and tested smart windows for office rooms in Oakland, CA, and the National Renewable Energy Laboratories (NREL) has ongoing research on developing prototypes of vertically integrated, photovoltaic powered electrochromic displays. In a different application, the optical change of chromogenic materials due to proton intercalation is promising for hydrogen sensor applications and has been demonstrated by NREL researchers using $\mathrm{WO}_{3}$ as a molecular hydrogen sensor. The Dow Chemical Co.'s COMMOTION technology, based on printed electrochromic inks, has been specifically developed for use in promotional products including smart label and inexpensive display applications. COMMOTION has already been used by a U.K. retailer, Marks \& Spencer, for an animated greeting card application. NTERA's NanoChromics technology allows for fabrication of flexible display devices benefiting from high surface area of nanostructured metal oxides blended with organic electrochromes. Finally, Cidetec of North Spain has introduced polymer-based electrochromic false nails, probably the oddest application the field has encountered. A significant amount of information about electrochromism and related applications can be found on the World Wide Web. ${ }^{9}$

Conjugated polymers, while not as developed as the other systems, promise high contrast ratios, rapid response times, and long lifetimes for use in EC display technology. In this paper we address the ability to physically structure polymer-based electrochromic devices (ECDs) and exert control over their EC responses. Several patterning methods for polymeric ECDs are discussed, and examples of multicolor displays are shown, which are made possible through patterning of electrode surfaces.

\section{Fundamentals of Electrochromism}

There are three main types of electrochromic materials in terms of their electronically accessible optical states. The first type includes materials with at least one colored and one bleached state. These materials are especially useful for absorption/transmission-type device applications such as smart windows and optical shutters. Typical examples of this area are metal oxides, viologens, and polymers such as poly(3,4-ethylenedioxythiophene) (PEDOT). A second class of materials consists of electrochromes with two distinctive colored states. These EC materials lack a transmissive state but are useful for display-type applications where different colors are desired in different redox states. Polythiophene is a good example of this type, where the thin films of this polymer switch from red to blue upon oxidation. A third class includes the growing interest in the electrochromic field, where more than two color states are accessible depending on the redox state of the material. This is the area where conjugated polymers have found the most interest due to their versatility for making blends, laminates, and copolymers. Addition- ally, there are inherently multicolor EC polymers such as PANI or poly(3,4-propylenedioxypyrrole) (PProDOP). We will discuss these multicolored polymers in detail in the Color Control section. However, before we switch to a more detailed discussion of polymer electrochromism, here we outline some of the important parameters in identifying and characterizing the electrochromic materials.

Electrochromic Contrast. Electrochromic contrast is probably the most important factor in evaluating an electrochromic material. It is often reported as a percent transmittance change $(\Delta \% T)$ at a specified wavelength where the electrochromic material has the highest optical contrast. For some applications, it is more useful to report a contrast over a specified range rather than a single wavelength. To obtain an overall electrochromic contrast, measuring the relative luminance change provides more realistic contrast values since it offers a perspective on the transmissivity of a material as it relates to the human eye perception of transmittance over the entire visible spectrum. ${ }^{10,11}$ The light source used is calibrated taking into account the sensitivity of the human eye to different wavelengths.

Coloration Efficiency. The coloration efficiency (also referred to as electrochromic efficiency) is a practical tool to measure the power requirements of an electrochromic material. In essence, it determines the amount of optical density change $(\Delta \mathrm{OD})$ induced as a function of the injected/ejected electronic charge $\left(Q_{\mathrm{d}}\right)$, i.e., the amount of charge necessary to produce the optical change. It is given by the equation

$$
\eta=(\Delta \mathrm{OD}) / Q_{\mathrm{d}}=\log \left[T_{\mathrm{b}} / T_{\mathrm{c}}\right] / Q_{\mathrm{d}}
$$

where $\eta\left(\mathrm{cm}^{2} / \mathrm{C}\right)$ is the coloration efficiency at a given $\lambda$ and $T_{\mathrm{b}}$ and $T_{\mathrm{c}}$ are the bleached and colored transmittance values, respectively. The relationship between $\eta$ and the charge injected to the EC material can be used to evaluate the reaction coordinate of the coloration process, or the $\eta$ values can be reported at a specific degree of coloration for practical purposes.

Switching Speed. Switching speed is often reported as the time required for the coloring/bleaching process of an EC material. It is important especially for applications such as dynamic displays and switchable mirrors. The switching speed of electrochromic materials is dependent on several factors such as the ionic conductivity of the electrolyte, accessibility of the ions to the electroactive sites (ion diffusion in thin films), magnitude of the applied potential, film thickness, and morphology of the thin film. Today subsecond switching rates are easily attained using polymers and composites containing small organic electrochromes.

Stability. Electrochromic stability is usually associated with electrochemical stability since the degradation of the active redox couple results in the loss of electrochromic contrast and hence the performance of the EC material. Common degradation paths include irreversible oxidation or reduction at extreme potentials, $i R$ loss of the electrode or the electrolyte, side reactions due to the presence of water or oxygen in the cell, and heat release due to the resistive parts in the system. Although current reports include switching stabilities of up to $10^{6}$ cycles without significance performance loss, the lack of durability (especially compared to LCDs) is 
still an important drawback for commercialization of ECDs. Defect-free processing of thin films, careful charge balance of the electroactive components, and airfree sealing of devices are important factors for longterm operation of ECDs.

Optical Memory. One of the benefits of using an electrochromic material in a display as opposed to a light-emitting material is its optical memory (also called open-circuit memory), which is defined as the time the material retains its absorption state after the electric field is removed. In solution-based electrochromic systems such as viologens, the colored state quickly bleaches upon termination of current due to the diffusion of soluble electrochromes away from the electrodes (a phenomenon called self-erasing). In solid-state ECDs, where the electrochromes are adhered to electrodes, the electrochromic memory can be as long as days or weeks with no further current required. In reality, however, ECDs may require small refreshing charges to maintain the charge state because side reactions or short circuits change the desired color.

\section{Origin of Electrochromism in Conjugated Polymers}

Conjugated polymers such as derivatives of $\mathrm{PPy}, \mathrm{PTh}$, and PANI display electrochromism in thin film form. Alkoxy-substituted PTh derivatives, such as PEDOT, have been investigated due to their ease of synthesis, high chemical stabilities in the oxidatively doped state, and high optical contrast values between redox states. ${ }^{12}$ Electrochromism in conjugated polymers occurs through changes in the conjugated polymer's $\pi$-electronic character accompanied by reversible insertion and extraction of ions through the polymer film upon electrochemical oxidation and reduction. In their neutral (insulating) states, these polymers show semiconducting behavior with an energy gap $\left(E_{\mathrm{g}}\right)$ between the valence band (HOMO) and the conduction band (LUMO). Upon electrochemical or chemical doping ("p-doping" for oxidation and "n-doping" for reduction), the band structure of the neutral polymer is modified, generating lower energy intraband transitions and creation of charged carriers (polarons and bipolarons), which are responsible for increased conductivity and optical modulation.

The doping process, and the resultant optical changes in conjugated polymers, can most vividly be illustrated through spectroelectrochemical experiments such as the one shown in Figure 1 for a thin film of poly(3,3-diethyl3,4-dihydro-2H-thieno[3,4-b][1,4]dioxepine) (PProDOT$\left.\mathrm{Et}_{2}\right)$. This polymer is purple-blue in the neutral state, and upon electrochemical oxidation switches to a transmissive sky blue in the oxidized (conducting) state. The neutral (colored) state of PProDOT-Et $t_{2}$ has a strong $\pi-\pi^{*}$ absorption in the visible region and a band gap of 1.7 $\mathrm{eV}\left(\lambda_{\max }=580 \mathrm{~nm}\right){ }_{.}^{13}$ Initial oxidation (p-doping) results in a new absorption band in the near-IR region $(\sim 900$ $\mathrm{nm}$ ), forming at the expense of the $\pi-\pi^{*}$ transition, which is attributed to polarons (radical cations) generated along the polymer chain. Upon complete electrochemical oxidation, the $\pi-\pi^{*}$ transition and the polaron absorption are fully depleted, while a lower energy transition, peaked in the near-IR beyond the range of the spectrophotometer, increases. This absorption is

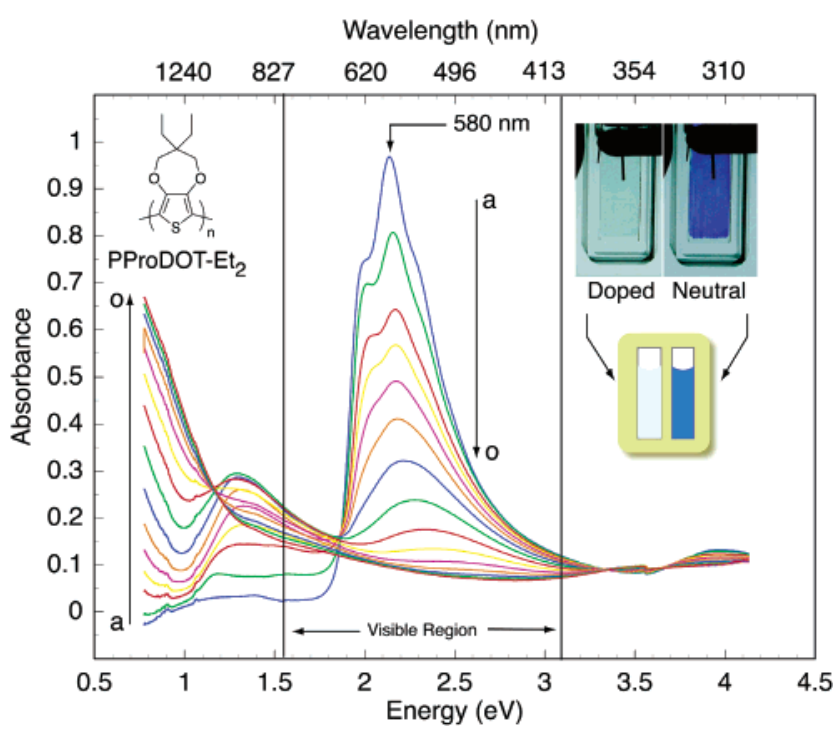

Figure 1. Spectroelectrochemistry of a PProDOT-Et $t_{2}$ film on ITO/glass at applied potentials between (a) $-0.1 \mathrm{~V}$ and (o) +0.9 $\mathrm{V}$ vs $\mathrm{Ag} / \mathrm{Ag}^{+}$with $50 \mathrm{mV}$ increments. ${ }^{13}$ The inset shows photographs of the polymer film in its doped and neutral states. Below the photographs are shown the CIE 1931 Yxy color swatches of the corresponding states measured by in situ colorimetry.

assigned to the bipolaronic (dication) state of the conjugated polymer. Such optical and structural changes are reversible through repeated doping and dedoping over many redox cycles, making EC polymers potentially useful in applications for modulating transmissivity and color.

\section{Characterization of Electrochromic Polymers: Methods}

To gain a deeper understanding of the electrochromic processes in conjugated polymers, multiple characterization methods have been developed. As discussed in the previous section, spectroelectrochemistry has been commonly used to study the electrochromic processes in conjugated polymers. However, spectroelectrochemistry does not allow one to precisely define contrast ratios or switching speeds. Thus, we and others have developed several other methods such as in situ colorimetric analysis, ${ }^{14}$ reflectance analysis, ${ }^{15,16}$ composite coloration efficiency determination, ${ }^{17}$ slow coloration efficiency determination, ${ }^{18}$ and fast electrochromic switching experiments ${ }^{19,20}$ in addition to spectroelectrochemistry. Using these primary techniques, we learn much about electrochromism in conjugated polymers.

For any commercial electrochromic material, specific and reproducible color states and contrast ratios are required. Therefore, in situ colorimetric analysis is used as a means of precisely defining color and contrast ratios in electrochromic polymers. The colorimetric analysis experiment is based on a set of color coordinates, such as the CIE 1931 Yxy color space. ${ }^{10}$ In this color space, $Y$ corresponds to the brightness or luminance of a color (specifically the brightness of the transmitted light in a transmission experiment), whereas the $x y$ coordinate of a color defines its hue and saturation. The benefit of defining a color via colorimetry rather than by simply stating a $\lambda_{\max }$ is that the CIE system of colorimetry is 
based on a standard observer and thus takes into account the manner in which the human eye perceives color. Colorimetric analysis thus gives a precise and accurate description of color.

In a typical experiment, the light transmitted through a polymer film is analyzed by a colorimeter (e.g., Minolta CS 100), which yields Yxy values. Perhaps the most useful information found through colorimetric analysis is the relative luminance $(\% Y)$. Here the measured luminance value $(Y)$ is taken relative to that of a standard white illuminant. Calculating the difference between $\% Y$ values measured at various applied potentials yields a measure of the contrast ratio that takes into account all wavelengths of the visible spectrum and the nonlinear response of the human eye.

Reflectance analysis, where the absorbance of an EC polymer is measured through the reflected light, provides useful information for investigating the optical properties of thin films on reflective substrates such as gold, platinum, and ITO. In particular, diffuse reflectance data may provide valuable information about the surface topology of a film since they take into account the scattered light in addition to the angular (specular) reflected light. In situ reflection spectroelectroscopy methods, where the absorbance of an EC polymer is monitored at different oxidation states, have been used to characterize PANI, PEDOT, and PProDOT polymers. ${ }^{14 b, 15 b, 21}$

Recently, we have developed the composite coloration efficiency (CCE) to characterize the efficiency of electrochromic polymers. ${ }^{17} \mathrm{CCE}$ is a measure of the change in optical density of a material at $\lambda_{\max }$ relative to the total amount of injected/ejected charge. CCE is thus a measure of how much charge is required to affect bleaching or coloration in an EC material. The experiment is based on a tandem chronoabsorptometry/chronocoulometry method in which the transmission at $\lambda_{\max }$ is monitored along with the charge passed as a polymer film is switched between redox states. In a standard experiment, we calculate CCE at $95 \%$ of the maximum optical contrast. Once this $95 \%$ change is reached, little additional color change is perceivable to the naked eye, and the complications of indefinitely increasing background charges are thus avoided.

By comparing the CCE values for different polymers, we can learn much about the effect of polymer structure on electrochromic properties. For example, with a homologous series of poly(3,4-alkylenedioxythiophene) (PXDOT) derivatives, we were able to show that increasing the steric bulk of the alkylenedioxy ring results in larger CCE values. ${ }^{17}$ This can be attributed to a more open polymer film morphology induced by the more sterically demanding rings, which allows higher doping levels and thus higher contrast ratios, through suppression of the visible absorbance bands.

Rauh et al. measured coloration efficiency $(\eta)$ as a function of doping level by injecting a certain amount of charge into a polymer layer galvanostatically. ${ }^{18}$ The $\eta$ value is initially linear with injected charge and reaches a maximum. At higher doping levels, due to the saturation of $\% T$ values, $\eta$ values drop substantially, suggesting that charge-consuming side reactions take place. We have also used this method for our absorptive/ transmissive-type devices based on solution-processed
EC polymers and have observed a similar trend during the coloration/bleaching process. ${ }^{16}$

Another method of EC polymer characterization commonly used is the use of single-wavelength spectrophotometry to monitor switching speeds and contrast ratios at $\lambda_{\max }$. The experiment is performed using the same experimental setup as that in spectroelectrochemistry and serves as an informative complement. Here a film is stepped from a potential at which the polymer is neutral to a potential at which the polymer is fully doped. The percent transmittance at the $\lambda_{\max }$ of the neutral polymer is monitored as a function of time as the polymer is repeatedly switched. This experiment gives a quantitative measure of the speed with which a film is able to switch between states. As with CCE, it is found that polymer structures that favor a more open morphology give rise to higher contrast ratios and faster switching speeds.

\section{Multicolor Electrochromic Polymers: Color Control}

In the field of EC materials, one of the great strengths of conjugated polymers is the ability to tailor the EC properties via modification of the polymer structure. Through band gap control, one can vary the accessible color states in both the doped and neutral forms of the polymer. Numerous synthetic strategies exist for tuning the band gap of conjugated polymers..$^{22}$ In practice, this band gap control is achieved primarily through main chain and pendant group structural modification. In the simplest approach, substitution of the parent heterocycle is used to affect the band gap through induced steric or electronic effects. Homopolymerization of comonomers or copolymerization of distinct monomers also gives rise to a modification of main chain polymer structure and allows for an interesting combination of the properties supplied by each monomer unit. Additionally, conjugated polymers can be utilized in blends, ${ }^{23}$ laminates, ${ }^{24}$ or composites ${ }^{25}$ to affect the ultimate color exhibited by the material; however, here we shall only consider color control which derives directly from modification of the chemical structure of a conjugated polymer. Using PEDOT as a platform, several approaches have been used to produce a wide variety of multicolor, variable-gap electrochromic polymers. Two such methods, chemical modification of the monomer and copolymerization, have proven to be effective routes. Using PEDOT as the basis for multicolor EC polymers, below we discuss a few representative examples from the literature to illustrate other concepts of color control in conjugated polymers. This brief overview is not intended as an exhaustive review.

While soluble, processable EC polymers are starting to develop into potentially useful materials, ${ }^{16,26-28}$ electropolymerization has long been the mainstay of EC polymers. It is this route which has generated the greatest variety of structurally diverse EC polymers. Figure 2 shows 15 polymers as examples of how structural modification of the monomer repeat unit is used to tune the band gap and achieve multicolor electrochromic polymers through homopolymerization. Color swatches based on CIE 1931 color coordinates are given where available. PANI (1) has multiple colored forms depending on the oxidation state of the polymer 


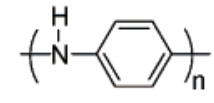

1<smiles>Cn1c(C(C)(C)C)ccc1C(C)(C)C</smiles><smiles>Cc1sc(-c2ccc3c(c2)c2cc(-c4sc(C(F)(F)F)c5c4OCCO5)ccc2n3C)c2c1OCCO2</smiles>

8

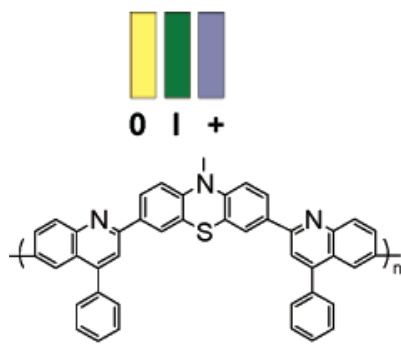

12

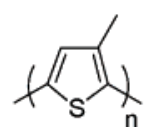

3

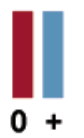

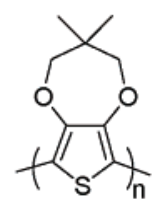

4

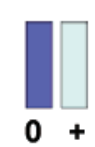<smiles>Cc1[nH]c(C)c2c1OCCO2</smiles>

5

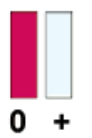<smiles>Cc1[nH]c(C)c2c1OCCCO2</smiles>

6

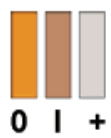

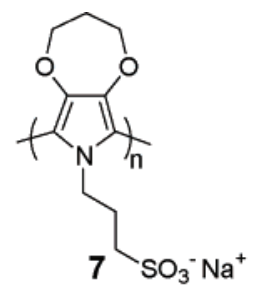

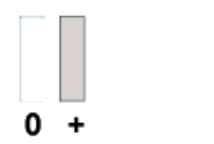

11
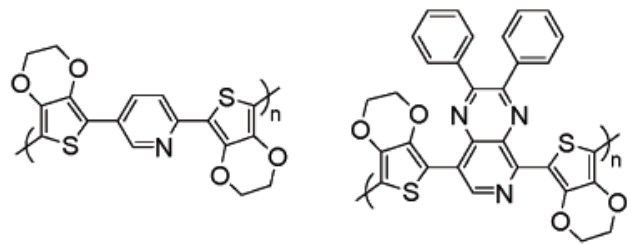

10
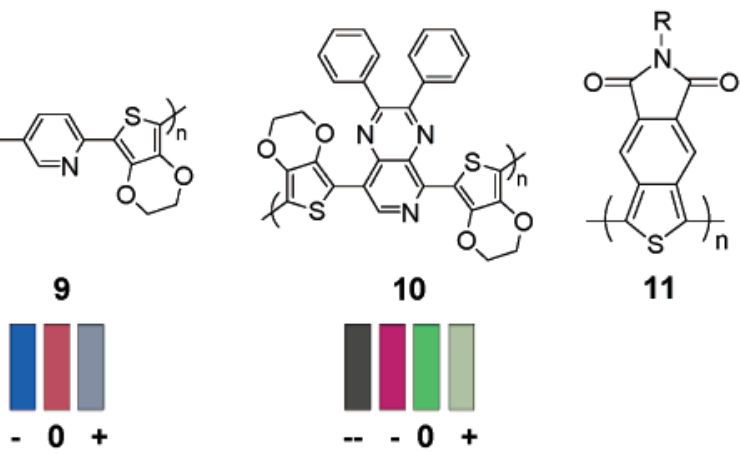

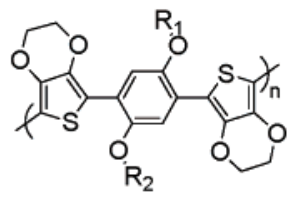

13

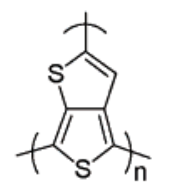

14

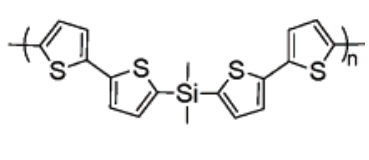

15

Figure 2. Representative electrochromic polymers. Color swatches are representations of thin films based on measured CIE 1931 Yxy color coordinates. Key: $0=$ neutral; $\mathrm{I}=$ intermediate; $+=$ oxidized; - and $--=$ reduced. Polymer structures and color swatches are adapted from refs 19 and $31-47$.

film which include leucoemeraldine (bright yellow), emeraldine (green), and pernigraniline (dark blue). ${ }^{8,29,30}$ $\mathrm{Poly}(N$-methylpyrrole) (PN-MePy) and poly(3-methylthiophene) (P3MeTh) $(\mathbf{2}, \mathbf{3})$ have shown stable and reversible electrochromism which later encouraged researchers to develop derivatized pyrrole- and thiophenebased polymers with improved electrochromic properties. Polymers 4-10 were developed to demonstrate the breadth of colors available in doped and neutral forms with relatively minimal change in the structures. PProDOT- $\mathrm{Me}_{2}$ (4) (poly(3,3-dimethyl-3,4-dihydro- $2 H$-thieno[3,4-b]dioxepine $)\left(E_{\mathrm{g}}=1.7 \mathrm{eV}\right)$ is a representative PXDOT derivative that shows little difference in color relative to PEDOT $\left(E_{\mathrm{g}}=1.6 \mathrm{eV}\right)$ as they are both cathodically coloring, deeply colored in their neutral states, and highly transmissive upon oxidation. ${ }^{19}$

PEDOP (5) [poly(3,4-ethylenedioxypyrrole)] is a representative PXDOP (poly(3,4-alkylenedioxypyrrole)) derivative. ${ }^{31,32}$ Here the electron-rich pyrrole gives rise to a material exhibiting a band gap of $2.0 \mathrm{eV}$ and thus a red neutral state and transmissive blue oxidized state. PProDOP (6) [poly(3,4-propylenedioxypyrrole)] illustrates how a slight structural modification of the monomer structure relative to that of PEDOP can result in a drastic change in the accessible color states. Here, PProDOP with a band gap of $2.2 \mathrm{eV}$ exhibits an orange neutral state, an intermediate brown state, and a gray/ blue oxidized state. Further modifying the repeat unit through $N$-substitution results in $N$-PrS-PProDOP (7) [poly( $N$-sulfonatopropoxy-ProDOP)]. Here the effect of $N$-substitution is to drastically increase the band gap to a value of $\geq 3.0 \mathrm{eV}$ as a result of unfavorable steric interactions between polymer repeat units based on the bulky sulfonatopropoxy substituent. As a result, this polymer is an anodically coloring material, changing from a completely transmissive and colorless neutral state to an absorbing light gray oxidized state..$^{33}$

PBEDOT- $N \mathrm{MeCz}$ (8) [poly(bis-EDOT- $N$-methylcarbazole) $]^{34}$ is a three-color electrochromic polymer formed from a multiring monomer (comonomer). Here the neutral polymer is a higher gap material $\left(E_{\mathrm{g}}=2.5 \mathrm{eV}\right)$, as the 3,6-linked incorporation of the carbazole into the main chain limits the extent of conjugation. Upon oxidative doping, this polymer shows two distinct redox processes and thus two additional color states, green at intermediate potentials (radical cation) and blue when fully oxidized (dication). PBEDOT-Pyr (9) [poly(bis-EDOT-pyridine)] and PBEDOT-PyrPyr (10) [poly(bis-EDOT-pyridopyrazine)] are also examples of multiring monomers or comonomers that exhibit multicolor electrochromism. ${ }^{35,36}$ Here, the donor-acceptor effect yields materials with low band gaps, which are capable of undergoing both $\mathrm{p}$ - and n-type doping. For PBEDOTPyr the band gap is $1.9 \mathrm{eV}$ due to the relatively weak pyridine acceptor. The polymer shows three distinct redox states (n-doped, neutral, and p-doped) and thus three colors. For PBEDOT-PyrPyr, the pyridopyrazine unit serves as a better acceptor than pyridine, and the 


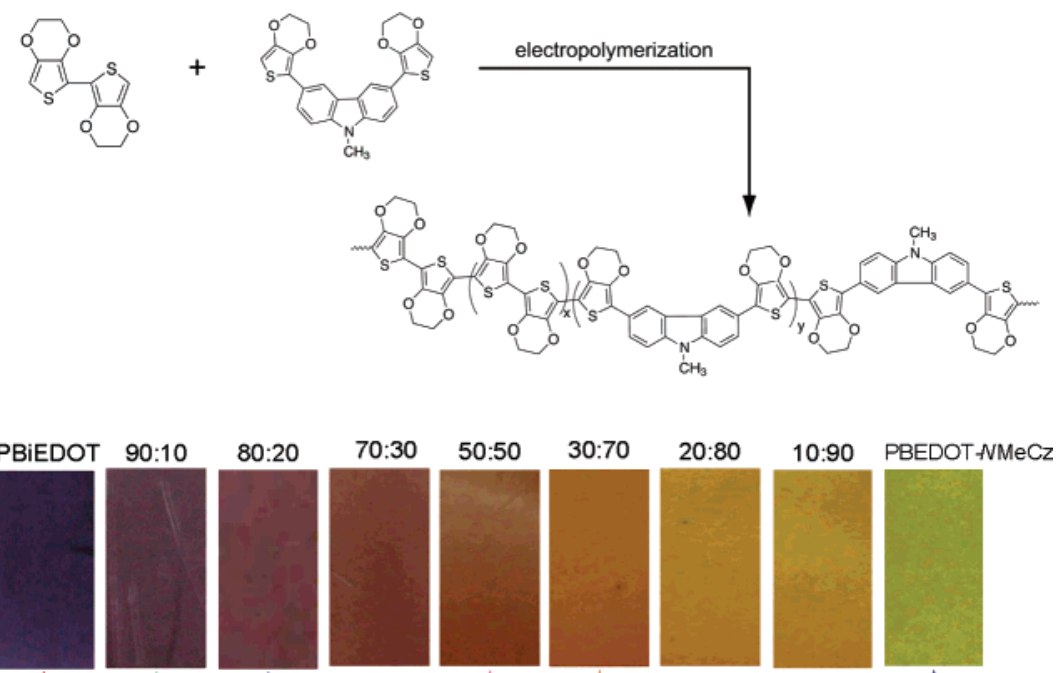

Figure 3. Top: Chemical scheme of the electropolymerization of BiEDOT (left) and BEDOT-NMeCz (right) to yield a representative random copolymer structure. ${ }^{49}$ Bottom: Photographs of neutral copolymer films on ITO/glass ranging from pure PBiEDOT on the left to pure PBEDOT-NMeCz on the right.

result is a significantly lower band gap polymer $(1.2 \mathrm{eV})$ and a four-color-state material with two n-doped states: a neutral state and a p-doped state.

Polymers 11-15 in Figure 2 are representative examples of other EC polymers from the literature. Poly(benzo[c]thiophene- $N$-2-ethylhexy-4,5-dicarboxylic imide) (EHI-PITN) (11) and its alternating copolymer with PEDOT are low-band-gap, n-type polymers which proved useful for their EC changes beyond the visible range in the near-IR region. ${ }^{28,37,38}$ Polymer 12 (PPTZPQ) [poly $\left(2,2^{\prime}\right.$-[10-methyl-3,7-phenothiazylene]-6,6'-bis [4phenylquinoline])] was described by Fungo et al.,39 which turns from yellow to red upon oxidation.

Polymer 13 \{(PBEDOT-B $\left.(\mathrm{OR})_{2}\right)$ [poly(bis-EDOTdialkoxybenzene)] $\}^{40-42}$ is an example of a bis-EDOTarylene polymer. This class of polymers was pioneered by the Reynolds' group and utilized by several other groups. ${ }^{40,43}$ In the case when a dialkoxybenzene is used as the arylene unit, the polymers exhibit low oxidation potentials, good stability to multiple switches, and two distinctly colored states. For the case of unsymmetrically substituted polymer $\mathbf{1 3}\left(\mathrm{R}_{1}=2\right.$-ethylhexyl, $\mathrm{R}_{2}=$ $\mathrm{CH}_{3}, E_{\mathrm{g}}=1.95 \mathrm{eV}$ ), a deep blue-purple neutral state is observed along with a nearly transparent light blue oxidized state. For the case of the symmetrical dialkoxybenzene analogues $\left(R_{1}=R_{2}=\right.$ heptyloxy or dodecyloxy), ${ }^{44}$ the band gap is found to be $1.95-2.0 \mathrm{eV}$ as well, but in these cases the polymers are pale red in the neutral state and deep blue in the oxidized state with a green color state observed at intermediate potentials. When the polymer is fully oxidized, it bears a transmissive blue state. This serves as further proof that slight variation of the repeat unit structure can drastically affect the colors exhibited by a polymer.

Polymers 14 and 15 represent two alternative approaches to the synthesis of EC polymers. Polymer $\mathbf{1 4}$ [poly(thieno[3,4-b]thiophene)] utilizes the polymerization of fused ring monomers to achieve an especially low band gap $(0.85 \mathrm{eV})$ electrochromic polymer. ${ }^{45,46}$ As a final example, polymer 15 illustrates an effective method of band gap control through the use of synthetically defined discrete conjugation length EC polymers. ${ }^{47}$ Here, the incorporation of a silicon linker between two bithiophenes limits the polymer conjugation length to four thiophene rings. As a result of this simple chemical modification, the polymer changes from a bright yellow neutral form to a dark green oxidized form as opposed to the normal red to blue electrochromism exhibited by polythiophene. Thus, as the previous examples have illustrated, by varying the chemical and electronic nature of the monomer, one can vary the color of the polymer and induce multicolor electrochromism.

Another method of color control which yields multicolor variable-gap conjugated polymers is based on the electrochemical copolymerization of known monomers. ${ }^{48}$ As was recently illustrated, ${ }^{49}$ this method is highly effective for producing multicolor electrochromic polymers as illustrated in Figure 3. Here, varying concentrations of BiEDOT and BEDOT- $\mathrm{NMeCz}$ are simultaneously oxidatively polymerized. These two monomers are chosen because they have roughly similar oxidation potentials ( 0.51 and $0.36 \mathrm{~V}$ vs $\mathrm{Ag} / \mathrm{Ag}^{+}$, respectively). As such, when a potential is applied, both monomers are oxidized and a random copolymer is formed as shown by the representative structure in Figure 3.

These copolymers of PBiEDOT and PBEDOT- $N \mathrm{MeCz}$ form color-tunable electrochromic materials with $\lambda_{\max }$ ranging from 424 to $580 \mathrm{~nm}$. Therefore, the polymer composition and thus the color are dependent on the relative concentrations of the two monomers. A continuous color gradient from pure PBiEDOT (dark blue), through red and orange intermediates, to pure PBEDOT- $N \mathrm{MeCz}$ (yellow) was obtained.

\section{Polymer Electrochromic Devices}

An ECD can be envisioned as an electrochemical cell where optical changes occur upon electrochemical reactions of two or more redox-active materials separated by an ionic conducting layer. Electrochromic switching of these devices is limited by diffusion of ions from one layer to another. ECDs based on inorganic electrochromes generally exhibit slow switching rates (multiple seconds) compared to liquid-crystal displays (LCDs), where optical changes occur through alignment of molecules under an applied electric field. However, 
LCDs depend on the viewing angle and are costly to process, and multiple colors cannot be obtained without addition of dyes. ${ }^{6}$ Efforts into making faster, more stable, and higher contrast ECDs have resulted in a remarkable increase in the number of patents and research papers, especially after the introduction of conjugated polymers as electrochromic materials. By judicious selection of electrochromic materials and by novel ECD designs, electrochromic switching rates of $1-10 \mathrm{~Hz}$ can be obtained. The long-term stability issues, often a major drawback for ECDs based on polymers, have now been overcome by introduction of air-stable polymers, novel polymer systems (blends, copolymers, composites, laminates, etc.), and new ionic media such as ionic liquids..$^{50}$ Coloration efficiency values of 500$3000 \mathrm{~cm}^{2} / \mathrm{C}$ can be attained due to the low charge requirements of the conjugated polymers. The availability of many solution-processible polymers has eased the fabrication of large-area ECDs. Adapting the currently available patterning methods, microstructured ECDs have emerged. Here we review some of the most recent ECD systems based on the conjugated polymers and counterparts. More information on ECDs from metal oxides and small organic molecules can be found elsewhere. ${ }^{51}$

Absorption/Transmission ECDs. An absorption/ transmission-type ECD operates by reversible switching of an EC material between a colored (absorptive) and a transmissive (bleached) state on a transparent, conducting substrate. To achieve high contrast values in such a device, two complementary polymers are used, namely, a cathodically coloring polymer and an anodically coloring polymer, deposited onto transparent electrodes (e.g., ITO/glass, ITO/PET, or PEDOT-PSS/PET), and separated by an electrolyte (viscous gel or solid) to allow ion transport as shown in Figure 4a.

The anodically coloring polymer is usually a highband-gap polymer and appears transmissive in the neutral state. Upon oxidation, it colors absorbing light in the visible region. The cathodically coloring polymer has a low band gap and is colored in its neutral (undoped) state, becoming transmissive upon oxidation. Therefore, when both polymers are sandwiched together and an external voltage is applied, the device switches between a colored state and a transmissive state. This type of device design has found use in applications such as smart windows and optical shutters.

Conjugated polymers have been used in several types of ECD systems as anodically and/or cathodically coloring materials. PANI was commonly used as a complementary electrode with metal oxide electrochromic layers such as $\mathrm{WO}_{3} .{ }^{52}$ Leventis et al. used surfaceconfined composites of polypyrrole-Prussian Blue (anodically coloring) with a polyviologen as the cathodically coloring material..$^{53}$ Other examples include a dodecyl sulfate derivatized $\mathrm{PPy}$ coupled with $\mathrm{WO}_{3}$ to obtain a $\Delta \% T$ of $\sim 45 \%$ at $600 \mathrm{~nm}^{54}$ and a charge-balanced device of $\mathrm{WO}_{3}$ using poly(3,4-ethylenedioxythiophene-didodecyloxybenzene) (PEB) as the cathodically coloring polymer. ${ }^{18}$ Most recently, Tung and Ho used a PEDOT/ Prussian Blue couple to fabricate ECDs with coloration efficiency values of $\sim 300 \mathrm{~cm}^{2} / \mathrm{C} .{ }^{55}$

ECDs with all polymer electrochromes have been widely studied in the literature. Using ITO-coated
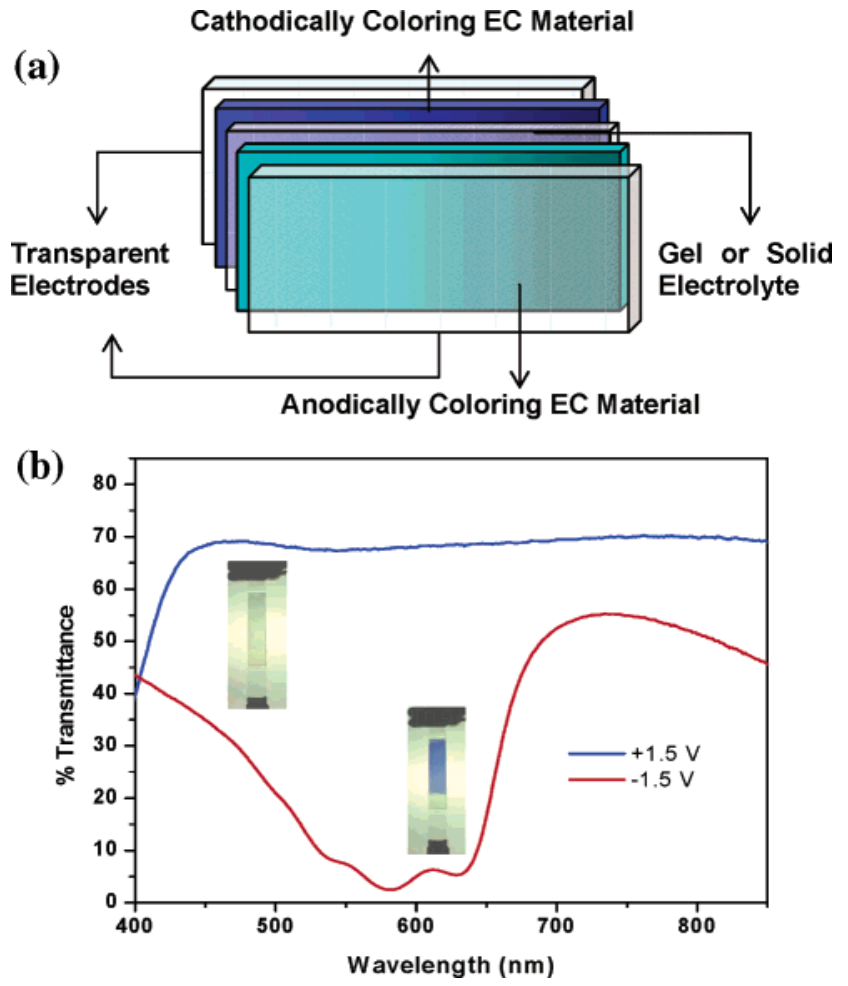

Figure 4. (a) Schematic representation of an absorption/ transmission-type device. (b) Percent transmittance spectra of an absorption/transmission electrochromic device composed of the complementary polymers PProDOT- $\mathrm{Me}_{2}$ and $N$-PrSPProDOP. The voltage applied to switch the system is \pm 1.5 $\mathrm{V}$. The inset shows photographs of the device in the bleached and colored states. Reprinted from ref 59. Copyright 2002 American Chemical Society.

plastic substrates, many complementarily colored polymers have been investigated to obtain flexible and polymer-based ECDs. ${ }^{56}$ DeLongChamp and Hammond have used the layer-by-layer assembly method to deposit soluble EC polymers electrostatically on ITO electrodes and have fabricated complementary ECDs by pairing PEDOT and PANI. ${ }^{57}$ The layer-by-layer electrostatic adsorption of a sulfonated derivative of PEDOT has been investigated by our group, where the multilayer thin films exhibit a fast and reversible redox switching behavior in aqueous media. ${ }^{58}$

We have optimized the visible region absorption of two polymers so that they could give an optimized contrast ratio in a window-type ECD when they operate in a complementary fashion. ${ }^{59} \mathrm{PProDOT}-\mathrm{Me}_{2}$ is used as the cathodically coloring polymer due to its outstanding contrast in the visible region $(\Delta \% T=78 \%$ at $580 \mathrm{~nm})$. A high-band-gap, pyrrole-based polymer, $N$-PrS-PProDOP $\left(E_{\mathrm{g}}=2.9 \mathrm{eV}\right)$, was used as the complementary anodically coloring polymer. Figure $4 \mathrm{~b}$ shows the percent transmittance spectra and photographs of the ECD in its two extreme states (colored and transmissive). The device possesses a $\Delta \% T$ of $68 \%$ at $580 \mathrm{~nm}\left(\lambda_{\max }\right.$ for the device) and switches between states in $\sim 0.5 \mathrm{~s}$ under a bias voltage of $\pm 1.5 \mathrm{~V}$. In this way, high-contrast ECDs based on conjugated polymers can be reproducibly constructed.

As discussed earlier, colorimetric analysis is a useful method for investigating the electrochromic properties of ECDs, providing information on color and relative luminance. In addition, this is a valuable method for 

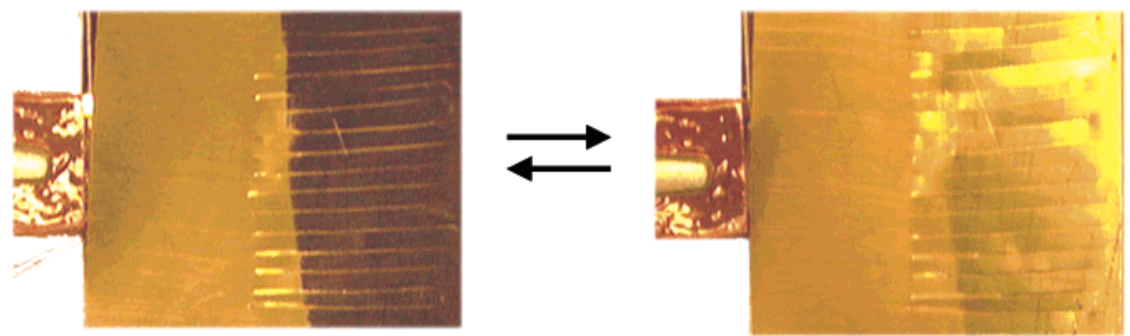

Figure 5. Electrochromic switching of PProDOT-Me $\mathrm{H}_{2}$ in a surface-active ECD. Left: neutral, colored state. Right: oxidized, reflective state. Reprinted with permission from ref 69. Copyright 2001 Wiley-VCH.

measuring the stability of ECDs upon repeated redox switching. Specifically, the initial change in relative luminance $(\Delta \% Y)$ of the PProDOT-Me $/ N$-PrS-PProDOP device is $55 \%$. The long-term stability of this luminance change was monitored over the course of several days during repeated switching between states. Initially a $10 \%$ loss in contrast was observed during the first 500 switches. However, after this conditioning period, contrast degradation slowed, with the ECD losing only $4 \%$ of its contrast after an additional 20000 switches, demonstrating the potential for high stability of conjugated polymer electrochromic devices.

Two examples of truly all polymer ECDs have been recently reported where the ITO layer has been replaced by highly conducting PEDOT-PSS to achieve allpolymer ECDs. PEDOT-PSS films are processed from an aqueous dispersion which is commercially produced in large quantities by Bayer A.G. (Baytron-P) and AgfaGevaert. Researchers from Linköping University and Acreo have combined an electrochemical transistor with an ECD to build an active matrix paper display. ${ }^{60} \mathrm{We}$ have constructed ECDs using different complementary pairs of EC polymers on PEDOT-PSS-coated transparent plastic electrodes and have demonstrated that PEDOT-PSS is an excellent replacement for ITO ${ }^{61}$

Reflective ECDs. Electrochromism is not limited to visible color changes, but can be extended to encompass materials that exhibit radiation modulation in the nearinfrared, mid-infrared, and microwave regions. ${ }^{62,63}$ This has provided the impetus for developing ECDs that can operate at longer wavelengths, beyond the visible region, with long lifetimes and fast redox switching times. Bessiere et al. have recently reported an IR modulator ECD using powder hydrates of tungsten oxide embedded in a plastic matrix with contrast values of $30-50 \% .^{64}$ Other IR-modulating devices based on $\mathrm{WO}_{3}$ include those of Hale et al. ${ }^{65}$ and Franke et al. ${ }^{66}$ Polymer-based devices comprising PANI-CSA as the active EC material have been used for thermal emissivity control in the near-IR and mid-IR regions (2.5$20 \mu \mathrm{m}) .{ }^{67}$ PEDOT's IR electrochromism has been studied by Pages et al. in broad-band ECDs using porous gold electrodes, where they optimized the pore size and gold thickness for reflectance analysis. ${ }^{15 a}$

To characterize the infrared EC properties of the polymers synthesized in our laboratories, we have employed a flexible, outward facing, reflective device platform originally developed by Bennett and Chandrasekhar. ${ }^{68} \mathrm{~A}$ device was constructed by electrosynthesizing PProDOT- $\mathrm{Me}_{2}$ as the surface-active EC polymer (due to its outstanding contrast ratio and high stability) onto a slitted (slit separation $\sim 1-2 \mathrm{~mm}$ ) goldcoated Mylar reflective conducting substrate as shown in Figure $5{ }^{69}$ As this film is switched from its neutral, colored state to its oxidized, bleached state, a color change of the ECD from absorptive blue to reflective gold takes place in $3 \mathrm{~s}$. In the visible region, EC switching yielded a reflectance contrast ratio of $55 \%$ at $600 \mathrm{~nm}$. In the near-IR region, the contrast ratio was as high as $90 \%$ at $1.8 \mu \mathrm{m}$.

Patterned ECDs. For EC polymer display technology to evolve toward higher definition devices, new methods for active material deposition must be developed. Therefore, a significant amount of attention has been directed toward information displays that require a high degree of visible color contrast. Typical device construction is based on sandwich-type configurations, similar to the ones discussed earlier, where at least one of the electrodes is transparent. An emerging facet of ECD construction pursued by researchers is the metalization of a surface via patterning methods. This is useful since it allows the combination of at least two polymers at both large (centimeter) and small (micrometer) scales that can display a set of colors on a surface, or be averaged by the human visual perception by color mixing. Moreover, metalization to form contact electrodes may be performed on ionic-permeable materials to develop reflective/absorptive surfaces with especially rapid switching rates. Chandrasekhar et al. have used porous electrodes to investigate PANI-based flexible devices for spacecraft thermal control applications with contrast values of $40-50 \%$ in the mid-IR region. ${ }^{63} \mathrm{We}$ have recently utilized metal-vapor deposition and the line-patterning process developed by MacDiarmid et al. ${ }^{70}$ to deposit conjugated EC polymers for the construction of novel ECDs. Other patterning methods employed include screen printing, microcontact printing $(\mu \mathrm{CP})$, and inkjet printing. ${ }^{71}$ Here we present a brief review of some of the patterning techniques that are used to fabricate electrodes for polymer ECDs. Many other patterning and printing techniques might be applied in the future depending on the needs for resolution, cost, and accessibility.

Metal-Vapor Deposition. ECDs have been constructed employing porous polycarbonate membranes that have been metalized with a thin layer of gold. Specific gold patterns have been deposited by attaching a physical mask to the naked substrate prior to metal deposition. Typically, a $50 \mathrm{~nm}$ layer of gold is sufficient to yield a well-adhered shiny gold electrode, while maintaining the porous nature of the flexible electrode. This point is important given that high surface reflectivity is required to afford a useful visible/near-IR contrast, and porosity is necessary to facilitate ion flux in the final device. Conjugated polymers are electrosynthesized directly onto the gold surface or can be sprayed and 


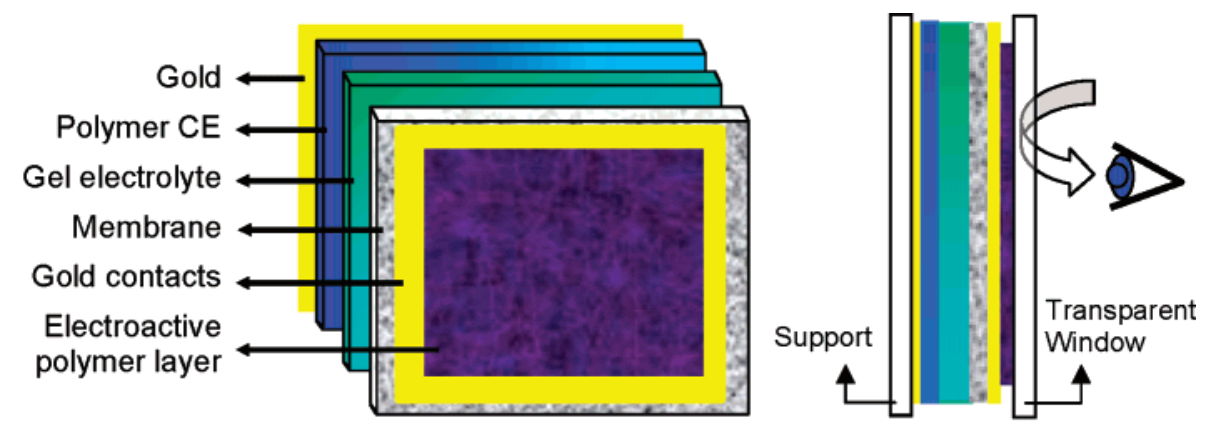

(b)

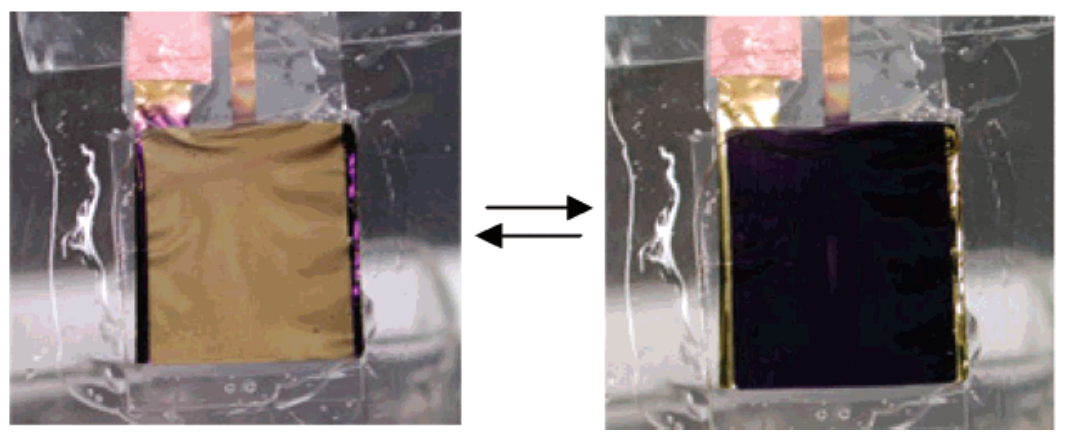

Figure 6. (a) A reflective device scheme using porous electrodes. (b) Electrochromic switching of a PProDOT-Me 2 device on a gold-coated porous membrane. Left: oxidized film at $+1.0 \mathrm{~V}$. Right: neutral film at $-1.0 \mathrm{~V}$.

solution coated. ${ }^{16}$ Using the electrodeposition method, multiple polymers can be incorporated into the same array-type device by first depositing one polymer, and after washing with monomer-free electrolyte solution, electrodepositing the second polymer onto the array. Figure 6a schematically shows the construction of a 1.5 $\times 2.0 \mathrm{~cm}$ single-"pixel" device by placing an electroactive polymer-coated counter electrode, coated with a gel electrolyte (PMMA/propylene carbonate based), to the back of the patterned membrane. Thus, the patterned porous membrane has the active layer facing outward. Here the importance of a porous electrode becomes obvious since it allows the EC material to face outward while still having the ability to switch between color states, similar to the slitted devices discussed earlier. On the basis of this architecture, we have constructed porous-type ECDs with PProDOT-Me $\mathrm{Me}_{2}$ as the active layer as shown in Figure 6b. ${ }^{72}$ The device shown in Figure $6 \mathrm{~b}$ switches between a dark-blue (neutral polymer) absorbing state and a very transmissive (oxidized polymer) state, revealing the highly reflective gold surface. Dramatic improvements in switching speed were observed in these second-generation devices relative to the slitted devices simply by modifying the nature of the conducting substrate. The $3 \mathrm{~cm}^{2}$ device pictured switches between the absorptive and reflective states in subsecond time frames with a 95\% optical switch attainable in $200 \mathrm{~ms}$. Rapid switching speeds are a direct result of the porous nature of the gold layer, which allows a high flux of charge-compensating ions to pass between electrodes, which in turn leads to high doping levels in the PProDOT-Me $\mathrm{Me}_{2}$ active layer. Small power consumption is typical for such conjugated polymer ECDs to maintain their colored or bleached states. When the device is assembled under noninert conditions, a stability test reveals that this device is able to switch hundreds of thousands of times over the course of several days. Thus, we expect device lifetimes to increase after inert atmosphere construction conditions are employed.

More complex device construction is attained easily as we exploit the enhanced properties of conjugated polymers deposited onto metalized porous membranes. To demonstrate this, we have assembled a $2 \times 2$ pixeltype array device consisting of two conjugated polymers that display unique visible absorptions (blue and red) in the neutral state while maintaining the ability to switch to a very transmissive oxidized state. PEDOT and PBEDOT-B $(\mathrm{OR})_{2}$ were electrodeposited onto the porous membrane and then switched with a bias voltage of $\pm 1 \mathrm{~V}$. As shown in Figure 7, at a bias of $-1 \mathrm{~V}$, PEDOT is blue while PBEDOT-B $(\mathrm{OR})_{2}$ is red. When the bias is reversed and both polymers are fully oxidized, both polymers switch to a highly transmissive state, exposing the reflective gold surface.

This simple concept of color and contrast in a primitive display evokes conceptual thinking of higher resolution pixel devices and provides the basis for color control, through structural modification of the EC polymer, in the construction of lateral ECDs on flexible substrates.

Line Patterning. Line patterning (first reported by MacDiarmid et al. ${ }^{70}$ ) is an excellent method to build finestructured electrodes on surfaces such as plastic or paper. Metalized electrodes in the sub-millimeter range have been prepared by initially printing a black ink pattern "negative" onto a flexible substrate. The substrate, together with the ink pattern, is then metalized with gold ${ }^{73}$ via an electroless deposition method. Following metalization, the ink negative is removed by sonication in toluene to produce a patterned electrode. Examples of a few of the line-patterned electrodes constructed in our laboratories are shown in Figure 8. 


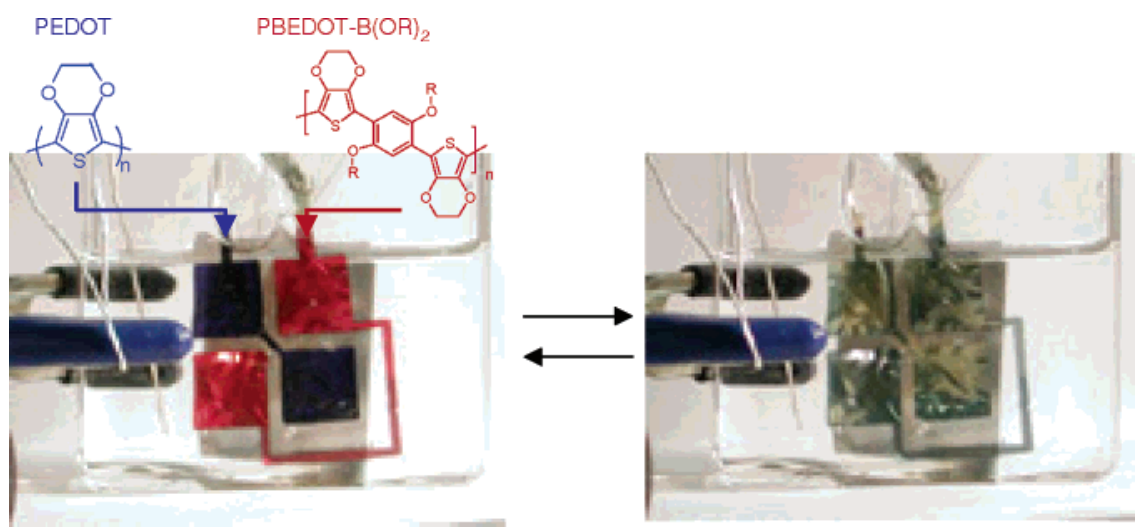

Figure 7. Photographs of EC switching of PEDOT and PBEDOT-B $(\mathrm{OR})_{2}$ on a $2 \times 2$ pixel gold/membrane electrode. Left: both polymers in their neutral (colored) states. Right: polymers in their oxidized (bleached) states.
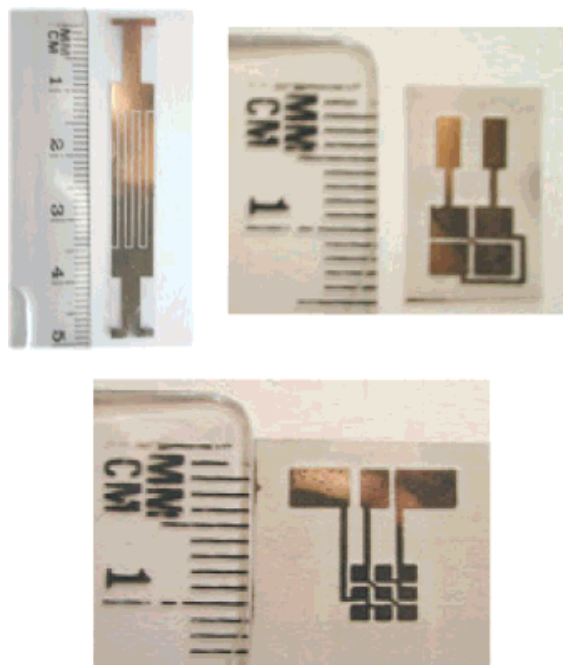

Figure 8. Photographs of gold-patterned electrodes obtained by the line-patterning method. To left: interdigitated electrode. Top right: $2 \times 2$ pixels. Bottom: $3 \times 3$ pixels.

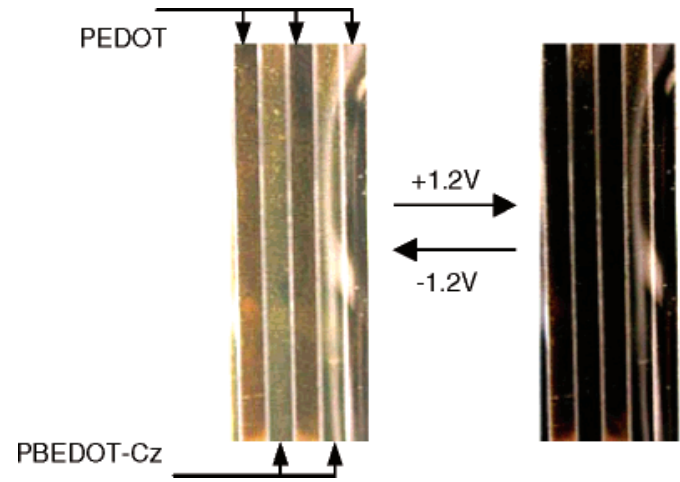

Figure 9. EC switching of two complementary polymers (PEDOT and PBEDOT-Cz) on an interdigitated, line-patterned electrode. Left: bleached, reflective state. Right: colored, absorptive state.

Laterally configured dual-polymer ECDs have subsequently been constructed utilizing this method. As shown in Figure 9, PEDOT (cathodically coloring) and PBEDOT-Cz (anodically coloring) were separately electrosynthesized on alternating lines of an interdigitated electrode device.

After electrodeposition, the EC polymer active layer was coated with an ionically conductive gel, followed by encapsulation. By applying opposite bias voltages to each polymer, a high-contrast, laterally configured ECD is achieved. The ultimate switching characteristics of the lateral device can be tuned through further architectural engineering of the metalized pattern.

Screen Printing. Screen printing is an additive patterning method where the desired material is selectively deposited through a template mask with resolution values of $\sim 10-100 \mu \mathrm{m}$. Introduced for electroactive polymers by Garnier et al. for printing electronic circuitry of polymer FETs, ${ }^{74}$ there are only a few examples of this technique for ECD applications. Coleman et al. ${ }^{75}$ used this method to print electrical contacts for finely patterned ECDs. Brotherston et al. ${ }^{76}$ have demonstrated checkerboard- and stripe-patterned ECDs comprising color-mixing PEDOT and $\mathrm{V}_{2} \mathrm{O}_{5}$ as electrochromic materials. Andersson et al. ${ }^{60}$ of Acreo have combined an organic transistor with a display ECD all based on organic materials using screen printing. In this work, solution-processible PEDOT has been printed on a paper both as the transistor component and the active EC material to produce smart pixels.

\section{Perspective and Outlook}

The past few years have seen an immense set of developments in the properties of EC polymers and their application to multiple device configurations. Using a variable monomer structure, or by adjusting the composition of copolymers, composites, and blends, the varied color states of EC polymers can be tuned across the visible spectrum and applied to wavelengths outside of the visible region. The facts that an EC polymer presents a minimum of two distinct color states, can be continuously modulated as a function of the applied voltage, and in some instances can present multiple distinct color states offer a significant amount of flexibility for display- and window-type devices. As solventand electrolyte-swollen films that are directly attached to transparent conducting or reflective metallic surfaces, the electroactive centers are directly addressable and can be switched quite rapidly. Using appropriate electrode geometries, rapidly switching polymers with subsecond response times can be envisioned. The repeated switching stabilities measured, typically on the order of $10^{4}$ to $10^{5}$ deep cycles, provide an area where improvement is desired. As these limitations are likely due to loss of electroactivity and/or adhesion at the electrode surface, methods to make the electrode materials more compatible (e.g., surface functionalization) with the EC polymer structure are being implemented. 
As the redox states are set by applied potential and are physically separated from one another, polymer-based ECDs can present a significant level of electrochromic memory. For example, initial studies on reflective devices show that a $1 \mathrm{~V} / 1 \mathrm{~s}$ refreshing pulse can retain the polymer in both the colored and transmitting states with especially long resting periods (many minutes) at open circuit.

Acknowledgment. We gratefully acknowledge the AFOSR (Grant No. F49620-03-1-0091) the ARO (MURI Program No. DAAD19-99-1-0316, EIC Grant No. DACA39-99-C-0004), the ONR, and the University of Pennsylvania Materials Science Laboratory (NSF Award No. DMR-32598) for the funding of our electrochromic polymer and line-patterning research.

\section{References}

(1) Monk, P. M. S.; Mortimer R. J.; Rosseinsky, D. R. Electrochromism: Fundamentals and Applications; VCH: Weinheim, Germany, 1995.

(2) Platt, J. R. J. Chem. Phys. 1961, 34, 862

(3) (a) Deb, S. K. Appl. Opt., Suppl. 1969, No. 3, 192-195. (b) Deb, S. K. Philos. Mag. 1973, 27, 801.

(4) (a) Granqvist, C. G. Sol. Energy Mater. Sol. Cells 2000, 60, 201. (b) Granqvist, C. G.; Avendano, E.; Azens, A. Thin Solid Films 2003, 442, 201. (c) Faughnan, B. W.; Crandall, R. S.; Heyman, P. M. RCA Rev. 1975, 36, 177.

(5) Habib, M. A. Electrochem. Transition 1992, 51.

(6) Somani, P. R.; Radhakrishnan, S. Mater. Chem. Phys. 2003, 77, 117.

(7) (a) Bach, U.; Corr, D.; Lupo, D.; Pichot, F.; Ryan, M. Adv. Mater. 2002, 14, 845. (b) Corr, D.; Bach, U.; Fay, D.; Kinsella, M.; McAtamney, C.; O’Reilly, F.; Rao, S. N.; Stobie, N. Solid State Ionics 2003, 165, 315.

(8) Mortimer, R. J. Electrochim. Acta 1999, 44, 2971.

(9) See the following Web sites: http://www.chem.ufl.edu/ reynolds, http://www.gentex.com, http://www.donnelly.com, http:// www.dynamircorp.com/, http://eetd.lbl.gov/btp, http:// www.sage-ec.com, http://www.nrel.gov/buildings/windows, http:// www.ntera.com, http://www.acreo.se, and http://www.cidetec.es

(10) Wyszecki, G.; Stiles, W. S. Color Science; Wiley: New York, 1982

(11) Berns, R. S.; Billmeyer, F. W.; Saltzman, M. Billmeyer and Saltzman's principles of color technology, 3rd ed.; Wiley: New York, 2000.

(12) Groenendaal, L. B.; Jonas, F.; Freitag, D.; Pielartzik, H.; Reynolds, J. R. Adv. Mater. 2000, 12, 481 .

(13) Gaupp, C. L.; Welsh, D. M.; Reynolds, J. R. Macromol. Rapid Commun. 2002, 23, 885

(14) (a) Thompson, B. C.; Schottland, P.; Zong, K.; Reynolds, J. R. Chem. Mater. 2000, 12, 1563. (b) Hyodo, K.; Omae, M. J. Electroanal. Chem. 1990, 292, 93.

(15) (a) Pages, H.; Topart, P.; Lemordant, D. Electrochim. Acta 2001 , 46, 2137. (b) Chang, Y. H.; Lee, K.; Kiebooms, R.; Aleshin, A.; Heeger, A. J. Synth. Met. 1999, 105, 203.

(16) Cirpan, A.; Argun, A. A.; Grenier, C. R. G.; Reeves, B. D.; Reynolds, J. R. J. Mater. Chem. 2003, 13, 2422.

(17) Gaupp, C. L.; Welsh, D. M.; Rauh, R. D.; Reynolds, J. R. Chem. Mater. 2002, 14, 3964.

(18) Rauh, R. D.; Wang, F.; Reynolds, J. R.; Meeker, D. L. Electrochim. Acta 2001, 46, 2023.

(19) Welsh, D. M.; Kumar, A.; Meijer, E. W.; Reynolds, J. R. Adv. Mater. 1999, 11, 1379

(20) Kumar, A.; Welsh, D. M.; Morvant, M. C.; Piroux, F.; Abboud, K. A.; Reynolds, J. R. Chem. Mater. 1998, 10, 896

(21) Hwang, J.; Tanner, D. B.; Schwendeman, I.; Reynolds, J. R. Phys. Rev. B 2003, 67, 115205 .

(22) Roncali, J. Chem. Rev. 1997, 97, 173.

(23) Meeker, D. L.; Mudigonda, D. S. K.; Osborn, J. M.; Loveday, D. C.; Ferraris, J. P. Macromolecules 1998, 31, 2943.

(24) Boehme, J. L.; Mudigonda, D. S. K.; Ferraris, J. P. Chem. Mater. 2001, 13, 4469.

(25) DeLongchamp, D. M.; Kastantin, M. K.; Hammond, P. T. Chem. Mater. 2003, 15, 1575.

(26) Wang, F.; Wilson, M. S.; Rauh, R. D.; Schottland, P.; Thompson, B. C.; Reynolds, J. R. Macromolecules 2000, 33, 2083

(27) Welsh, D. M.; Kloeppner, L. J.; Madrigal, L.; Pinto, M. R.; Thompson, B. C.; Schanze, K. S.; Abboud, K. A.; Powell, D.; Reynolds, J. R. Macromolecules 2002, 35, 6517.

(28) Meng, H.; Tucker, D.; Chaffins, S.; Chen, Y.; Helgeson, R.; Dunn, B.; Wudl, F. Adv. Mater. 2003, 15, 146.
(29) Lacroix, J. C.; Kanazawa, K. K.; Diaz, A. J. Electrochem. Soc 1989, 136, 1308.

(30) Ching, J.-C.; MacDiarmid, A. G. Synth. Met. 1986, 13, 193.

(31) Gaupp, C. L., Zong, K., Schottland, P., Thompson, B. C., Reynolds, J. R. Macromolecules 2000, 33, 1132

(32) Schottland, P.; Zong, K.; Gaupp, C. L.; Thompson, B. C.; Thomas, C. A.; Giurgiu, I.; Hickman, R.; Abboud, K. A.; Reynolds, J. R. Macromolecules 2000, 33, 7051.

(33) Sonmez, G.; Schwendeman, I.; Schottland, P.; Zong, K.; Reynolds, J. R. Macromolecules 2003, 36, 639.

(34) Sotzing, G. A.; Reddinger, J. L.; Katritzky, A. R.; Soloducho, J.; Musgrave, R.; Reynolds, J. R. Chem. Mater. 1997, 9, 1578.

(35) Irvin, D. J.; DuBois, C. J., Jr.; Reynolds, J. R. Chem. Commun. $1999,2121$.

(36) DuBois, C. J.; Reynolds, J. R. Adv. Mater. 2002, 14, 1844.

(37) Meng, H.; Wudl, F. Macromolecules 2001, 34, 1810.

(38) Sonmez, G.; Meng, H.; Wudl, F. Chem. Mater. 2003, 15, 4923

(39) Fungo, F.; Jenekhe, S. A.; Bard, A. J. Chem. Mater. 2003, 15, 1264

(40) Sotzing, G. A.; Reynolds, J. R.; Steel, P. J. Chem. Mater. 1996, 8,882 .

(41) Irvin, J. A.; Schwendeman, I.; Lee, Y.; Abboud, K. A.; Reynolds, J. R. J. Polym. Sci., Part A: Polym. Chem. 2001, 39, 2164.

(42) Sonmez, G.; Meng, H.; Zhang, Q.; Wudl, F. Adv. Funct. Mater. 2003, 13, 726 .

(43) Pepitone, M. F.; Hardaker, S. S.; Gregory, R. V. Chem. Mater. 2003, 15, 557.

(44) Irvin, J. A.; Reynolds, J. R. Polymer 1998, 39, 2339

(45) Lee, K.; Sotzing, G. A. Macromolecules 2001, 34, 5746.

(46) Sotzing, G. A.; Lee, K. Macromolecules 2002, 35, 7281.

(47) Mudigonda, D. S. K.; Boehme, J. L.; Brotherson, I. D.; Meeker, D. L.; Ferraris, J. P. Chem. Mater. 2000, 12, 1508.

(48) Yohannes, T.; Carlberg, J. C.; Inganas, O.; Solomon, T. Synth. Met. 1997, 88, 15 .

(49) Gaupp, C. L.; Reynolds, J. R. Chem. Mater. 2003, 36, 6305.

(50) (a) Lu, W.; Fadeev, A. G.; Qi, B. H.; Mattes, B. R. J. Electrochem. Soc. 2004, 151, H33. (b) Lu, W.; Fadeev, A. G.; Qi, B. H.; Smela E.; Mattes, B. R.; Ding, J.; Spinks, G. M.; Mazurkiewicz, J.; Zhou, D. Z.; Wallace, G. G.; MacFarlane, D. R.; Forsyth, S. A.; Forsyth, M. Science 2002, 297, 983 .

(51) (a) Rosseinsky, D. R.; Mortimer, R. J. Adv. Mater. 2001, 13, 783. (b) Zhang, H.-T.; Subramanian, P.; Fussa-Rydel, O.; Bebel, J. C.; Hupp, J. T. Sol. Energy Mater. Sol. Cells 1992, 25, 315. (c) Cummins, D.; Boschloo, G.; Ryan, M.; Corr, D.; Rao, S. N.; Fitzmaurice, D. J. Phys. Chem. B 2000, 104, 11449.

(52) (a) Jelle, B. P.; Hagen, G.; Oedegaard, R. Electrochim. Acta 1992 37, 1377. (b) Jelle, B. P.; Hagen, G.; Birketveit, O. J. Appl. Electrochem. 1998, 28, 483. (c) Bernard, M.-C.; Goff, A. H.-L.; Zeng, W. Electrochim. Acta 1998, 44, 781. (d) Marcel, C.; Tarascon, J. M. Solid State Ionics 2001, 143, 89.

(53) Leventis, N.; Chung, Y. C. Chem. Mater. 1992, 4, 1415

(54) De Paoli, M.-A.; Zanelli, A.; Mastragostino, M.; Rocco, A. M. J. Electroanal. Chem. 1997, 435, 217.

(55) Tung, T.-S.; Ho, K.-C Proc.-Electrochem. Soc. 2003, 17, 254.

(56) (a) De Paoli, M.-A.; Nogueira, A. F.; Machado, D. A.; Longo, C Electrochim. Acta 2001, 46, 4243. (b) De Paoli, M. A.; CasalboreMiceli, G.; Girotto, E. M.; Gazotti, W. A. Electrochim. Acta 1999, 44, 2983. (c) Gazotti, W. A.; Casalbore-Miceli, G.; Geri, A.; Berlin, A.; De Paoli, M. A. Adv. Mater. 1998, 10, 1522. (d) Arbizzani, C.; Mastragostino, M.; Meneghello, L.; Morselli, M.; Zanelli, A. J. Appl. Electrochem. 1996, 26, 121. (e) Ferraris, J. P.; Henderson, C.; Torres, D.; Meeker, D. Synth. Met. 1995, 72, 147

(57) DeLongchamp, D.; Hammond, P. T. Adv. Mater. 2001, 13, 1455.

(58) Cutler, C. A.; Bouguettaya, M.; Reynolds, J. R. Adv. Mater. 2002, 14,684 .

(59) Schwendeman I.; Hickman R.; Sonmez G., Schottland P.; Zong K.; Welsh D. W.; Reynolds J. R. Chem. Mater. 2002, 14, 3118

(60) Andersson, P.; Nilsson, D.; Svensson, P.-O.; Chen, M.; Malmstrom, A.; Remonen, T.; Kugler, T.; Berggren, M. Adv. Mater. $2002,14,1460$.

(61) Argun, A. A.; Cirpan, A.; Reynolds, J. R. Adv. Mater. 2003, 15, 1338.

(62) Rauh, D. R. Electrochim. Acta 1999, 44, 3165.

(63) Chandrasekhar P.; Zay B. J.; Birur G. C.; Rawal S.; Pierson E. A.; Kauder L.; Swanson, T. Adv. Funct. Mater. 2002, 12, 95.

(64) (a) Bessiere, A.; Beluze, L.; Morcrette, M.; Lucas, V.; Viana, B.; Badot, J. C. Chem. Mater. 2003, 15, 2577. (b) Bessiere, A.; Marcel, C.; Morcrette, M.; Tarascon, J. M.; Lucas, V.; Viana, B.; Baffier, N. J. Appl. Phys. 2002, 91, 1589.

(65) Hale, J. S.; Woollam, J. A. Thin Solid Films 1999, 339, 174

(66) Franke, E. B.; Trimble, C. L.; Hale, J. S.; Schubert, M.; Woollam, J. A. J. Appl. Phys. 2000, 88, 5777 .

(67) (a) Topart, P.; Hourquebie, P. Thin Solid Films 1999, 352, 243. (b) Rose, T. L.; D’Antonio, S.; Jillson, M. H.; Kon, A. B.; Suresh R.; Wang, F. Synth. Met. 1997, 85, 1439. (c) Petit, N.; Gervais, F.; Buvat, P.; Hourquebie, P.; Topart, P. Eur. Phys. J. B 1999 12, 367. (d) Gervais, F.; Petit, N.; Popon, C.; Buvat, P. Eur. Phys. J. $B$ 2003, 31, 47 . 
(68) (a) Bennett, R. B.; Kokonasky, W. E.; Hannan, M. J.; Boxall, L. G. U.S. Patent 5,446,577, 1995. (b) Chandrasekhar, P. U.S. Patent 5,995,273, 1999.

(69) Schwendeman, I.; Hwang, J.; Welsh, D. W.; Tanner, D. B.; Reynolds, J. R. Adv. Mater. 2001, 13, 634.

(70) Hohnholz, D.; MacDiarmid, A. G. Synth. Met. 2001, 121, 1327.

(71) Holdcroft, S. Adv. Mater. 2001, 13, 1753.

(72) Aubert, P.-H.; Argun, A. A.; Cirpan, A.; Tanner, D. B.; Reynolds, J. R. Chem. Mater. 2004, 16, 2396.
(73) Morton, J. P. U.S. Patent 3,676,213, 1972.

(74) Garnier, F.; Hajlaoui, R.; Yassar, A. Science 1994, 265, 1684.

(75) Coleman, J. P.; Lynch, A. T.; Madhukar, P.; Wagenknecht, J. H. Sol. Energy Mater. Sol. Cells 1999, 56, 395.

(76) Brotherston, I. D.; Mudigonda, D. S. K.; Osborn, J. M.; Belk, J.; Chen, J.; Loveday, D. C.; Boehme, J. L.; Ferraris, J. P.; Meeker, D. L. Electrochim. Acta 1999, 44, 2993.

CM049669L 